\title{
FINITE ELEMENT ANALYSIS OF INCOMPRESSIBLE AND COMPRESSIBLE FLUID FLOWS WITH FREE SURFACES AND STRUCTURAL INTERACTIONS $\dagger$
}

\author{
K. J. Bathe, $\uparrow$ H. Zhang $\ddagger$ and M. H. Wang $\ddagger$ \\ †Massachusetts Institute of Technology, Cambridge, MA 02139, U.S.A. \\ ‡ADINA R \& D, Inc., Watertown, MA 02172, U.S.A.
}

\begin{abstract}
We present the current ADINA-F capabilities for fluid flow analysis. The fluid can be considered to be an incompressible or compressible medium. Free surfaces and the full interactions with structures in two- and three-dimensional conditions can be analyzed. The basic formulations and finite element discretizations used are described, the techniques for the solution of the finite element equations are briefly discussed, and the results of various demonstrative analyses are given.
\end{abstract}

\section{INTRODUCTION}

Tremendous advances have been accomplished during recent years in the analysis of general fluid flows and fluid flows with structural interactions. The use of finite elements has made it possible to analyze general fluid flows, with or without heat transfer, in complex geometries at high Reynolds and Peclet numbers, and to analyze the full interactions between structures and fluid flows.

The objective of this paper is to give an overall view of some capabilities for such analyses and to discuss various important issues pertaining to the solution techniques. The view is limited to the analysis capabilities that we have developed in the ADINA-F finite element program.

In the next section we present the general continuum mechanics equations used. The mathematical models of the fluids are based on Eulerian or arbitrary Lagrangian-Eulerian descriptions to allow direct coupling to structures represented by Lagrangian descriptions [1]. The fluids are modeled as compressible or incompressible media with various material laws.

In Section 3 we then describe our finite element discretization of the mathematical models. The finite elements satisfy the inf-sup condition for incompressible (or almost incompressible) behavior and appropriate upwinding techniques are employed for the analysis of high Reynolds and Peclet number flows. Our techniques for the automatic meshing are also mentioned. If structural interactions are considered, the fluid finite element mesh will, in general, be much finer than the structural mesh and the interface conditions between the two models are appropriately included in the analysis.

tSome of the original figures for this paper were generated with a color-producing terminal and submitted in color.
We briefly summarize in Section 4 our procedures for the solution of the finite element equations. Implicit and explicit integration can be used.

In Section 5, we then present some results obtained in the solution of various fluid flow problems with, in some cases, structural interactions. Finally, we conclude the presentation in Section 6 with some general remarks regarding our continuing developments in this area.

\section{MATHEMATICAL MODELS FOR FLUID FLOWS INCLUDING STRUCTURAL INTERACTIONS}

The first step in an analysis of a fluid flow (or structural problem) consists of defining the geometry, material data, and boundary conditions, see Fig. 1. The geometry may be created by the analyst or be imported from the data of a CAD program. Once this basic information is available, in a second step the analyst defines the mathematical model to be solved. The information processę in these steps is largely independent of the type of numerical method to be used in solving the mathematical model except that, of course, the available solution procedures should be able to solve the model.

\subsection{Incompressible fluid flow equations}

In many practical fluid flow situations, the fluid flows approximately as an incompressible medium. In such cases the mathematical model assuming total incompressibility is appropriately employed. The governing equations are, using a Cartesian reference frame $\left(x_{i}, i=1,2,3\right)$, index notation and the usual summation convention.

Momentum

$$
\rho\left(\frac{\partial v_{i}}{\partial t}+v_{i, j} v_{j}\right)=\tau_{i j, j}+f_{i}^{\mathbf{B}} .
$$




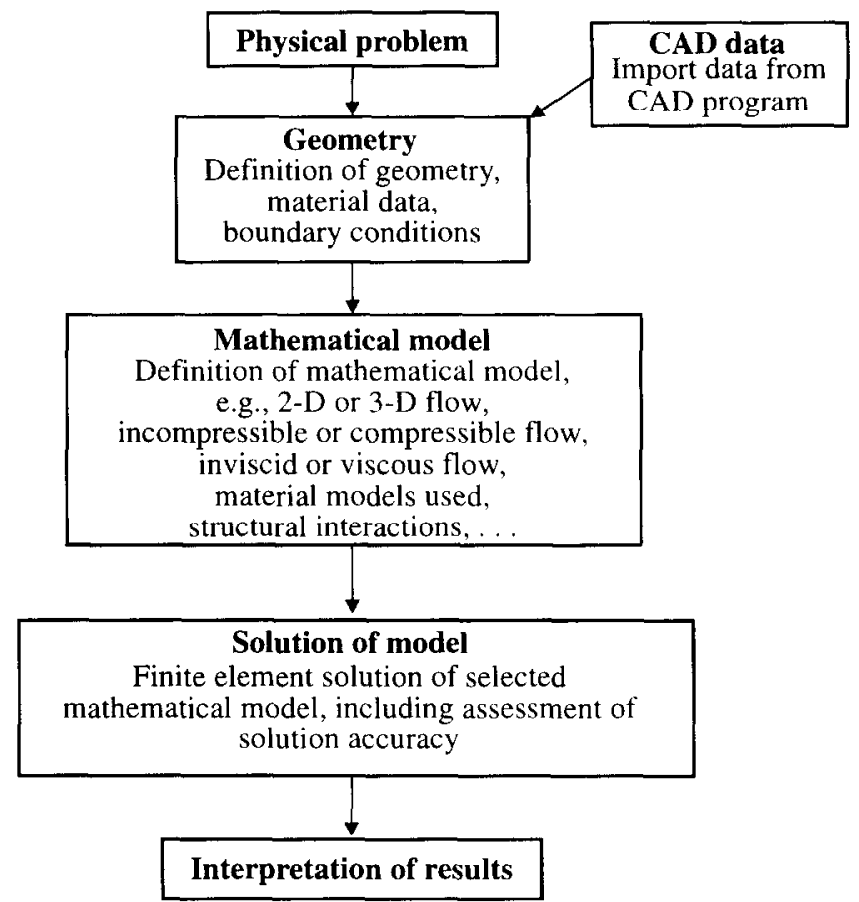

Fig. 1. Process of fluid flow analysis.

Constitutive

$$
\tau_{i j}=-p \delta_{i j}+2 \mu e_{i j}
$$

Continuity

$$
v_{i, i}=0
$$

Heat transfer

$$
\rho c_{p}\left(\frac{\partial \theta}{\partial t}+\theta_{, i} v_{i}\right)=\left(k \theta_{, i}\right)_{, i}+q^{\mathrm{B}}+2 \mu e_{i j} e_{i j},
$$

where we have, $v_{i}$, velocity of fluid flow in direction $x_{i} ; \rho$, mass density, constant; $\tau_{i j}$, components of stress tensor; $f_{i}^{\mathbf{B}}$, components of body force vector; $p$, pressure; $\delta_{i j}$, Kronecker delta; $\mu$, fluid viscosity, vclocity and temperature dependent; $e_{i j}$, components of velocity strain tensor $=1 / 2\left(v_{i, j}+v_{j, i}\right) ; c_{p}$, specific heat at constant pressure, temperature-dependent; $\theta$, temperature; $k$, thermal conductivity, temperaturedependent; $q^{\mathbf{B}}$, rate of heat generated per unit volume, temperature-dependent.

The boundary conditions corresponding to eqns (1)-(4) are:

- prescribed fluid velocities, $\bar{v}_{i}$, on the surface $S_{v}$

$$
v_{i}=\left.\bar{v}_{i}\right|_{S_{i}}
$$

- prescribed tractions, $f_{i}^{S}$, on the surface $S_{f}$

$$
\tau_{i j} n_{j}=\left.f_{i}^{S}\right|_{s_{f}}
$$

where $n_{j}$ are the components of the unit normal to the fluid surface and $f_{i}^{S}$ are the components of the traction vector;

- prescribed temperature, $\bar{\theta}$, on the surface $S_{\theta}$

$$
\theta=\left.\bar{\theta}\right|_{S_{\theta}}
$$

- prescribed heat flux into the surface $S_{q}$

$$
\left.k \frac{\partial \theta}{\partial n}\right|_{S_{q}}=q^{s}
$$

where $q^{S}$ is the heat flux into the body. We note that for eqns (5) and (6) $S_{v} \cup S_{f}=S, S_{v} \cap S_{f}=0$ and $S_{0} \cup S_{q}=S, S_{\theta} \cap S_{q}=0$, where $S$ is the total surface area of the fluid body.

The heat flow input in eqn (6b) comprises the effect of actually applied distributed heat flow and the effect of convection and radiation heat transfer.

In addition, we have for a free surface $S\left(x_{i}, t\right)$ the boundary conditions

$$
\frac{\partial S}{\partial t}+S_{, j} v_{j}=0
$$

and

$$
\tau_{i j} n_{j}=\left(p_{0}+\sigma\left(\frac{1}{R_{1}}+\frac{1}{R_{2}}\right)\right) n_{i}
$$

where $\sigma$ is the surface tension cocfficient, $p_{0}$ is the ambient pressure, and $R_{1}, R_{2}$ are the radii of curvature of the free surface. 
The above fluid flow equations correspond to laminar flow. Turbulence conditions can be represented using various turbulence models, including the $k-\epsilon$ model.

Equations (1)-(4) are in conservative form

$$
\frac{\partial \mathbf{U}}{\partial t}+\nabla \cdot(\mathbf{F}-\mathbf{G})=\mathbf{S}
$$

with

$$
\begin{gathered}
\mathbf{U}=\left[\begin{array}{c}
0 \\
\rho \mathbf{v} \\
\rho E
\end{array}\right] \\
\mathbf{F}=\left[\begin{array}{c}
\rho \mathbf{v} \\
\rho \mathbf{v} \\
\rho \mathbf{v} E-\boldsymbol{\tau} \cdot \mathbf{v}
\end{array}\right] \\
\mathbf{G}=\left[\begin{array}{c}
\mathbf{0} \\
\tau \\
-\mathbf{q}
\end{array}\right] \\
\mathbf{S}=\left[\begin{array}{c}
0 \\
0 \\
\mathbf{f}^{B} \\
\mathbf{f}^{B} \cdot \mathbf{v}+q^{B}
\end{array}\right]
\end{gathered}
$$

wherc, $E$, specific encrgy $=1 / 2 \mathbf{v} \cdot \mathbf{v}+e ; e$, internal energy; $\mathbf{q}$, heat flux $=-k \nabla \theta$.

In this mathematical model, we assume that $\mathrm{d} e=c_{v} \mathrm{~d} \theta$ where $c_{v}$ is the specific heat at constant volume, and $c_{v}=c_{p}$.

\subsection{Compressible fluid flow equations}

When the Mach number of the fluid velocities is larger than about 0.2 , the compressibility effects of the fluid need to be included. The governing equations of the mathematical model of a compressible fluid are, using index notation as in cqns (1)-(4).

Momentum

$$
\rho\left(\frac{\partial v_{i}}{\partial t}+v_{i, j} v_{j}\right)=\tau_{i j, j}+f_{i}^{\mathbf{B}} .
$$

Constitutive

$$
\tau_{i j}=-\left(p-\lambda v_{k, k}\right) \delta_{i j}+2 \mu e_{i j} .
$$

Continuity

$$
\frac{\partial \rho}{\partial t}+\left(\rho v_{i}\right)_{, i}=0
$$

\section{Energy}

$$
\frac{\partial(\rho E)}{\partial t}+\left(\rho v_{i} E-v_{j} \tau_{j i}+q_{i}\right)_{, i}=f_{i}^{\mathbf{B}} v_{i}+q^{\mathbf{B}},
$$

where now $\rho$ is of course not constant, and $\lambda$ is the second viscosity factor (in Stokes' hypothesis $\lambda=-2 / 3 \mu)$.
The conservative form of these equations is still

$$
\frac{\partial \mathbf{U}}{\partial t}+\nabla \cdot(\mathbf{F}-\mathbf{G})=\mathbf{S}
$$

where now, for the compressible flow conditions,

$$
\mathbf{U}=\left[\begin{array}{c}
\rho \\
\rho \mathbf{v} \\
\rho E
\end{array}\right]
$$

For use in Section 3.2 we also define

$$
\mathbf{F}^{*}=\left[\begin{array}{c}
\rho \mathbf{v} \\
\rho \mathbf{v} \mathbf{v}+p \mathbf{I} \\
\rho \mathbf{v} H
\end{array}\right] \quad \mathbf{G}^{*}=\left[\begin{array}{c}
\mathbf{0} \\
\bar{\tau} \\
\bar{\tau} \cdot \mathbf{v}-\mathbf{q}
\end{array}\right]
$$

where

$$
\bar{\tau}_{i j}=\hat{\imath} v_{k, k} \delta_{i j}+2 \mu e_{i j}
$$

and $H$ is the enthalpy, equal to $E+p / \rho$.

Comparing the governing equations of compressible fluid flow with those of the incompressible flow, we notice the commonalities, but for eqn (18) we need to use the equations of state

$$
\begin{aligned}
& \rho=\rho(p, \theta) \\
& e=e(p, \theta)
\end{aligned}
$$

which for a perfect gas give $\rho=p /\left(\left(c_{p}-c_{i}\right) \theta\right)$ and $e=c_{v} \theta$. However, a more general description as implied in eqns (22) and (23) is valuable (see Section 3.4).

The basic solution variables in the incompressible fluid flow equations are the unknown velocity, pressure and temperature. We also employ the same variables for the solution of the compressible fluid flow equations, and also the boundary conditions are then those given in eqns (5) and (6). A consequence of using the same solution variables to describe the incompressible and compressible fluid mathematical models, is that we can have in one finite element

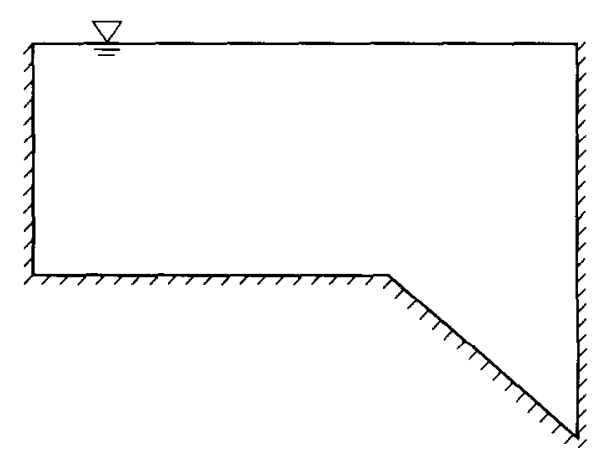

Fig. 2. Fluid in rigid cavity subjected to gravity loading (no-flow test). 
analysis regions modeled by both the compressible and incompressible fluid flow models.

\subsection{Arbitrary Lagrangian-Eulerian formulation}

For the analysis of fluid flows with structural interactions, it is effective to use an arbitrary LagrangianEulerian (ALE) formulation to describe the fluid flow $[2,3]$. This formulation can be directly coupled with a Lagrangian formulation for the structural response.
The essence of the ALE formulation is that the spatial position for the evaluation of the total time derivative of the variables of a fluid particle is not fixed in space, but is allowed to move. The motion is selected in the solution of the equations but does not necessarily correspond to the particle motion (which would be the case in a pure Lagrangian formulation).

Let $\mathbf{v}_{m}$ be the velocity of the spatial position used

(a)

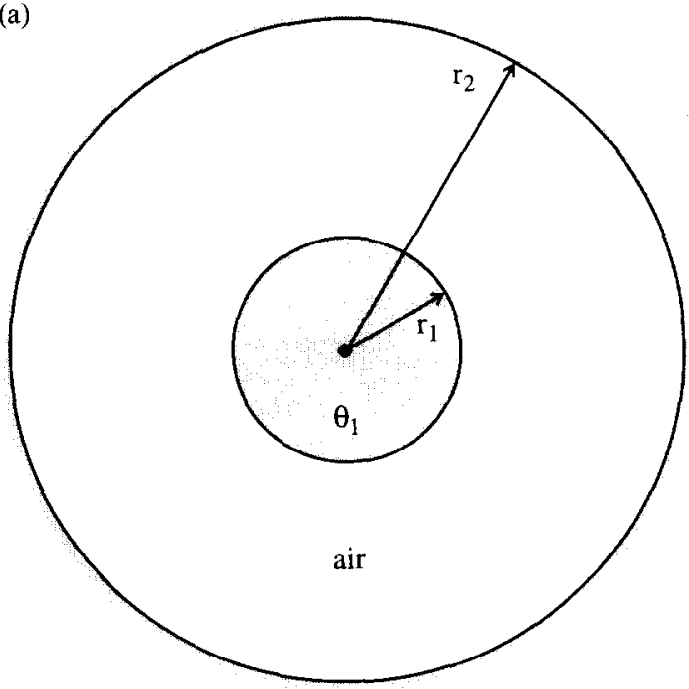

$\theta_{2}$

(b)

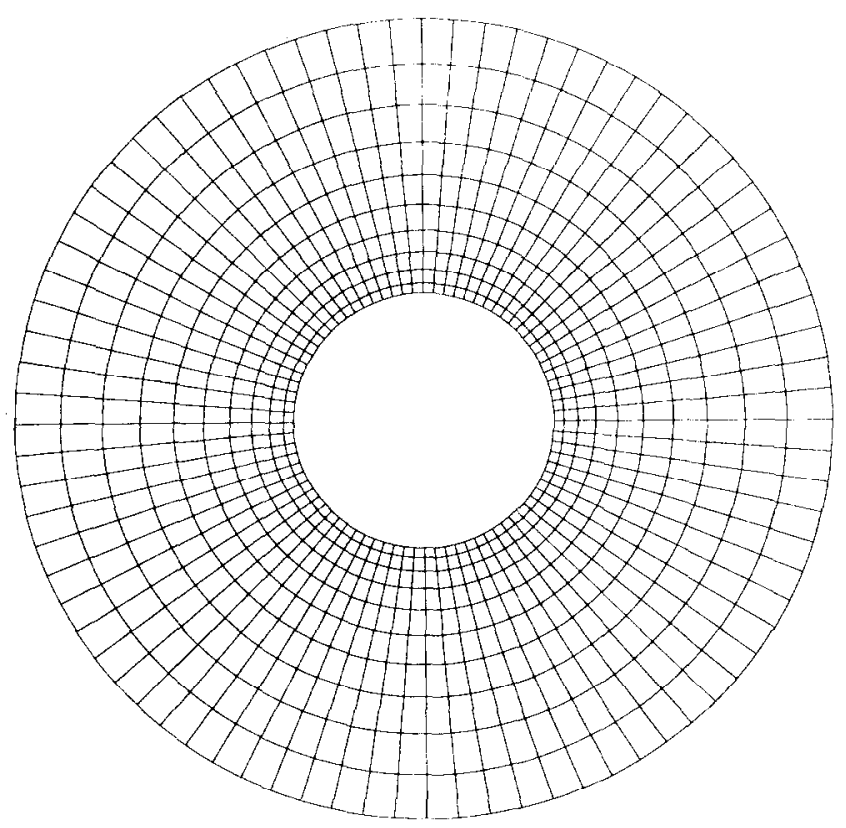

Fig. 3(a, b). Caption opposite. 
(c)
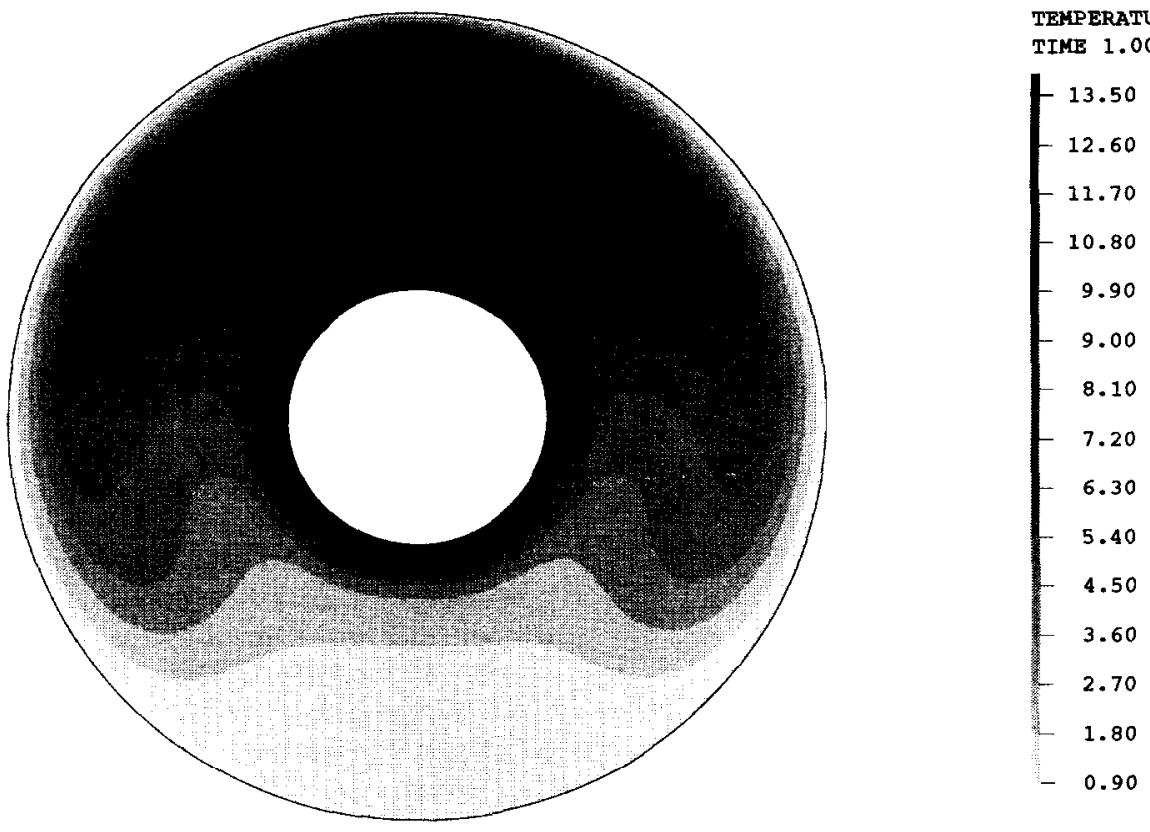

(d)
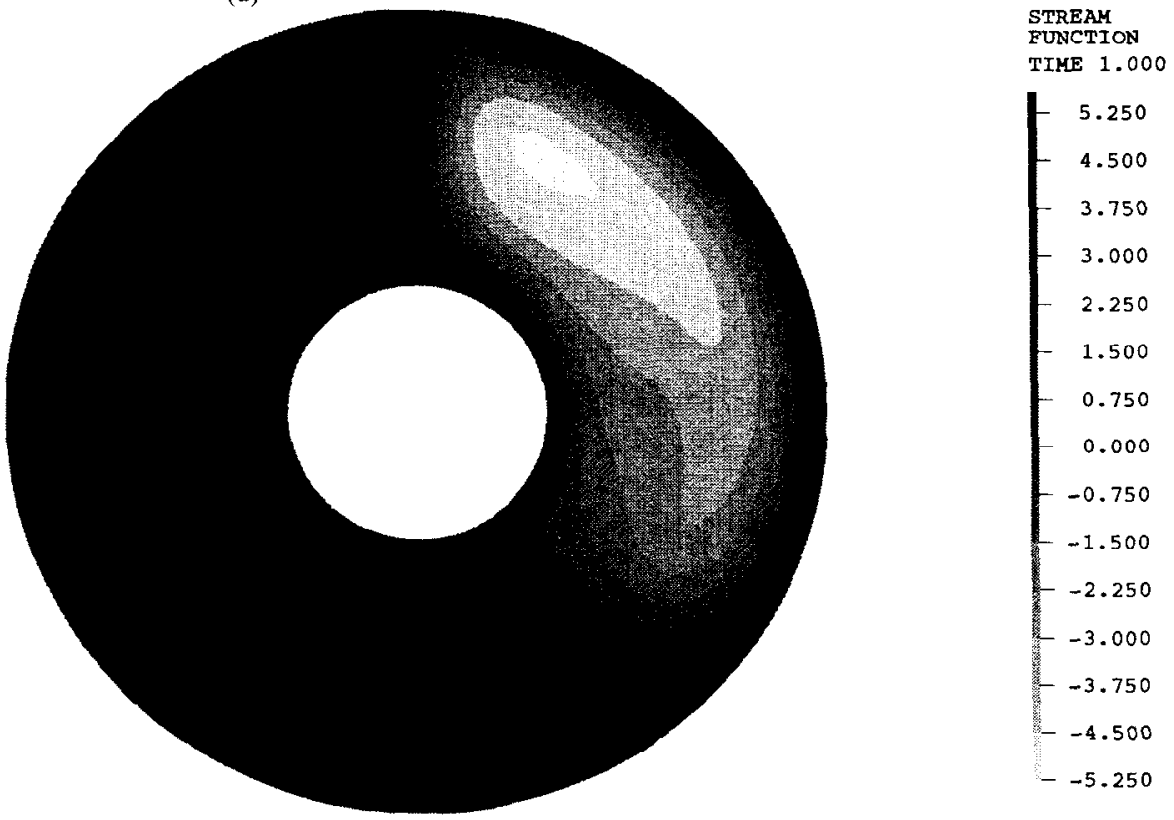

Fig. 3. Analysis of airflow between concentric cylinders due to temperature difference of cylinders. (a) Cylinders with analysis data $r_{2} / r_{1}=3.14, \theta_{1}-\theta_{2}=13^{\circ} \mathrm{C}$, Grashof number $=122,000$. (b) Finite element mesh used of 9/4-c elements. (c) Predicted isotherms. (d) Predicted streamlines.

for the time derivative of a variable, then the total time derivative of that variable is given by $[2,3]$

$$
\frac{\mathrm{d}(\cdot)}{\mathrm{d} t}=\frac{\delta(\cdot)}{\delta t}+\left(\mathbf{v}-\mathbf{v}_{m}\right) \cdot \nabla(\cdot)
$$

where $\delta(\cdot) / \delta t$ is the time derivative with respect to the spatial position. We use eqn (24) to express the total time derivatives in eqns (9) and (18), and prescribe the velocity $\mathbf{v}_{m}$ in the finite element solution process. 


\section{FINITE ELEMENT EQUATIONS}

The finite element solution of the continuum mechanics equations, given in the previous section, is achieved using the Galerkin variational procedure, with special techniques embedded, to be able to solve high Reynolds and Peclet number flows. The use of such techniques is necessary if the solution of complex fluid flows is to be achieved with reasonable and currently widely available computational resources.

\subsection{Finite elements used. Imporlance of inf-sup condition}

For low Reynolds and Peclet number flows the application of the usual Galerkin method yields, using eqns (9) and (18), for the weighting function $h_{i}$

$$
\begin{aligned}
\int_{V}\left(h_{i}\left(\frac{\partial \mathbf{U}}{\partial t}+\nabla \cdot \mathbf{F}-\mathbf{S}\right)+\nabla h_{i} \cdot \mathbf{G}\right) \mathrm{d} V \\
=\int_{S} h_{i} \mathbf{G}_{n} \mathrm{~d} S
\end{aligned}
$$

where $\mathbf{G}_{n}$ denotes the values in $\mathbf{G}$ in the direction of the unit normal $\mathbf{n}$ to the surface $S$ of the complete domain considered.

The weighting functions $h_{i}$ are of course given by the particular finite elements used in the discretizations.

The finite element used for incompressible fluid flow analysis should satisfy the inf-sup condition [1]. If this condition is satisfied, the element is optimal for the velocity and pressure interpolations employed; that is, the discretizations using the element are stable and have the "best" error bounds that we can expect for the interpolations used. For example, if element biquadratic velocities and a linear pressure are assumed, then the error in velocities is of $o\left(h^{3}\right)$ and the error in the stresses is $o\left(h^{2}\right)$.

Let $V_{h}$ be the finite element space of the velocities, with $\mathbf{v}_{h} \in V_{h}$, (and the subscript $h$ denoting the element "size" of the mesh considered) and $Q_{h}$ be the finite element space of the pressures, with $q_{h} \in Q_{h}$. Then the inf-sup condition is given by

$$
\inf _{q_{h} \in Q_{h}} \sup _{\mathbf{v}_{h} \in V_{h}} \frac{\int_{V} q_{h} \operatorname{div} \mathbf{v}_{h} \mathrm{~d} V}{\left\|q_{h}\right\|_{0}\left\|\mathbf{v}_{h}\right\|_{1}} \geqslant \beta>0
$$

where $\beta$ is a constant independent of the mesh [1]. Elements that satisfy the inf-sup condition are summarized in Refs [1] and [4].

While the use of elements that satisfy the inf-sup condition is necessary when the mathematical model is that of an incompressible fluid, such elements are also most effective in the analysis of compressible flows. Namely, in regions of low velocities, almost incompressible flow conditions may be encountered in a compressible flow solution.
We are using for the analysis of low Reynolds and Peclet number incompressible two-dimensional flows the biquadratic yelocity/temperature and bilinear pressure $\left(Q_{2}-Q_{1}\right.$ or $\left.9 / 4-c\right)$ quadrilateral element, and for high Reynolds and Peclet number incompressible flows and compressible twodimensional flows a triangular element with three corner nodes for velocity, temperature and pressure and an additional internal node for velocity (i.e. the 4/3-c element). For three-dimensional solutions, the corresponding three-dimensional elements are used These elements satisfy the inf-sup condition.

\subsection{Upwinding procedure for high Reynolds and} Peclet number flows

For high Reynolds and Peclet number flows, an upwinding procedure is embedded in the finite element equations. The essence of our formulation is to use the Galerkin procedure on the diffusive flux of the equations and a control volume type procedure on the convective flux. We have implemented the formulation for the triangular and tetrahedral element discretizations.

Considering inviscid compressible flow $\mathbf{G}^{*}=\mathbf{0}$ and let us define [5]

$$
\nabla \cdot \mathbf{F}^{*}=\left\|\nabla h_{k}\right\| \mathbf{A}_{k} \Delta_{i} \mathbf{U}_{k}
$$

where

$$
\begin{aligned}
\mathbf{A}_{k} & =\frac{\partial}{\partial \mathbf{U}}\left(\frac{\nabla h_{k} \cdot \mathbf{F}^{*}}{\left\|\nabla h_{k}\right\|}\right) \\
\Delta_{i} \mathbf{U}_{k} & =\mathbf{U}_{k}-\mathbf{U}_{i}
\end{aligned}
$$

and $\mathbf{U}_{k}$ and $\mathbf{U}_{i}$ are the values of $\mathbf{U}$ at nodal points $k$ and $i$ of the element considered.

The spectral decomposition of $\mathbf{A}_{k}$ is given by

$$
\mathbf{A}_{k}=\mathbf{P}_{k} \mathbf{D}_{k} \mathbf{P}_{k}^{-1}
$$

where $\mathbf{P}_{k}$ and $\mathbf{P}_{k}^{-1}$ store, respectively, the right and left eigenvectors, and $\mathbf{D}_{k}$ stores the corresponding eigenvalues. We apply the upwinding in the characteristic directions given by $\mathbf{P}_{k}$.

In viscous flow we use

$$
h_{i} \nabla \cdot \mathbf{F}^{*}+\nabla h_{i} \cdot \mathbf{G}^{*} \approx h_{i}\left\|\nabla h_{k}\right\|\left(\mathbf{A}_{k}-\mathbf{B}_{k}\right) \Delta_{i} \mathbf{U}_{k}
$$

where in the case of compressible flow we still have a decomposition similar to eqn (29)

$$
\mathbf{A}_{k}-\mathbf{B}_{k}=\tilde{\mathbf{P}}_{k} \tilde{\mathbf{D}}_{k} \tilde{\mathbf{P}}_{k}^{-1}
$$

However, in the case of incompressible flow we use directly the left-hand side in eqn (30) with $\mathbf{F}^{*}$ and $\mathbf{G}^{*}$ replaced by $\mathbf{F}$ and $\mathbf{G}$. In both cases the upwinding is applied to the flow directed through the element faces and can be implemented very effectively for the triangular and tetrahedral elements used. 
It is important to note that this "surface-directed" upwinding procedure does not change the basic Galerkin finite element equations (given in eqn (25)) when the Reynolds and Peclet numbers are small. Hence, in the case of small convective effects (small element Reynolds and Peclet numbers), the solution procedure reduces to solving the usual Galerkin equations.

\subsection{Meshing}

For the solution of a fluid flow problem, in general, a large number of finite elements is needed in the discretization. Since in certain regions a fine mesh may be required, whereas in other regions a rather coarse mesh may be sufficient, relatively small and large elements are generally used with transition zones.

(a)

\section{ADINA-F}
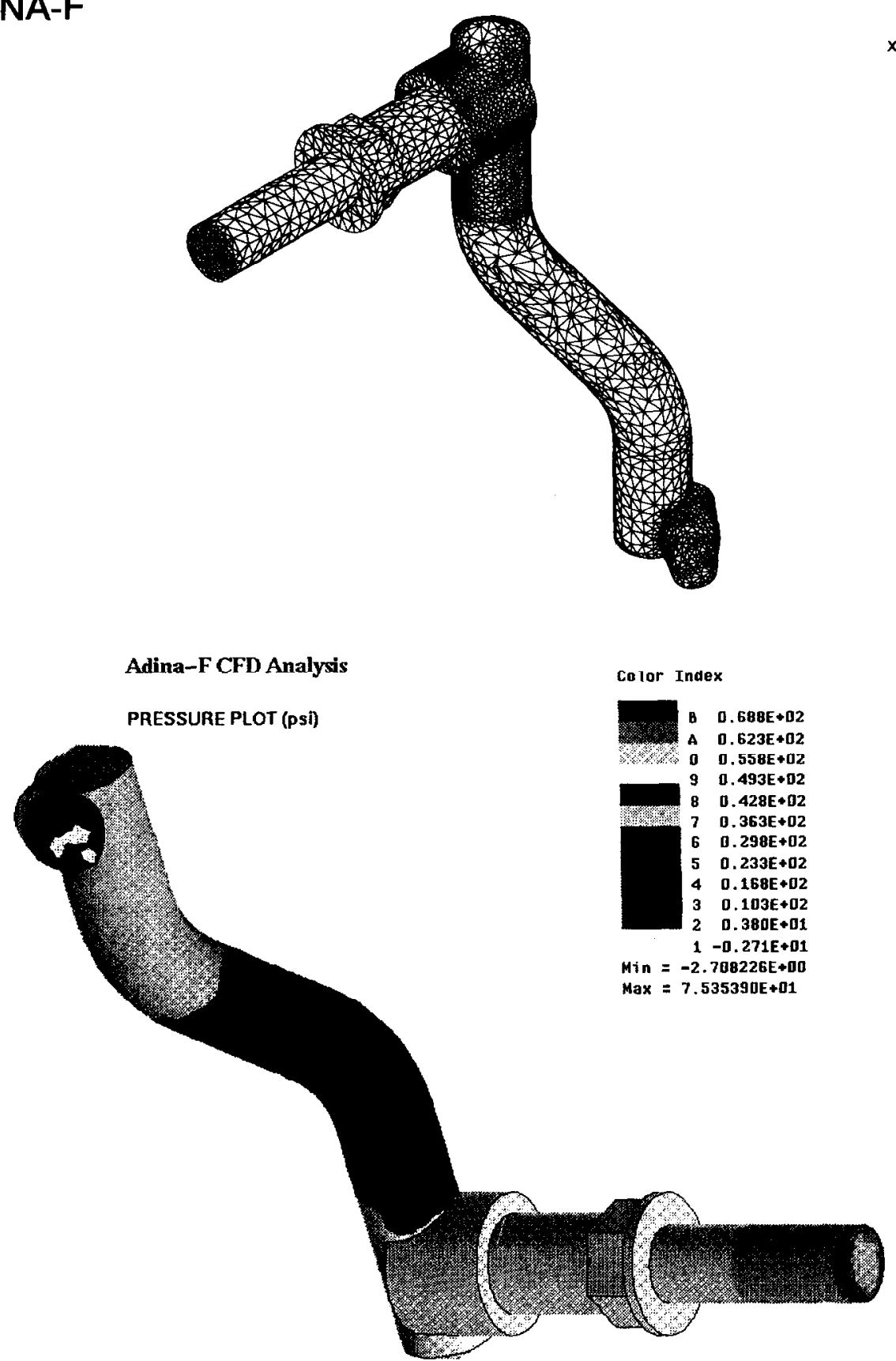

Fig. 4(a, b). Caption overleaf. 
(c)

\section{Adina-F CFD Analysis}

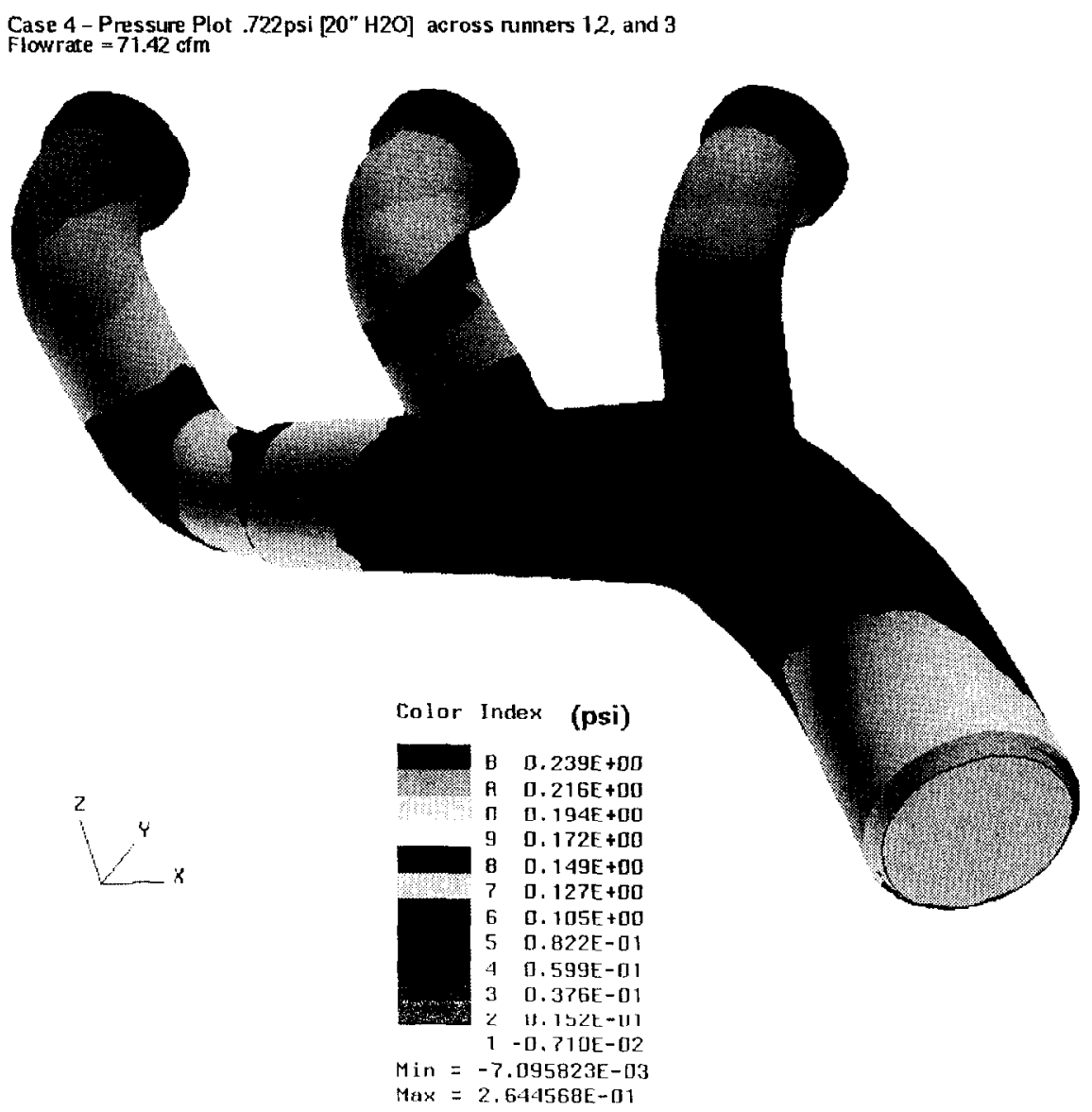

Fig. 4. Analysis of flow in pipe connections. (a) Finite element mesh used. (b) Predicted pressure distribution. (c) Predicted pressure distribution in additional pipe connection.

Our approach is to consider the complete geometry of the fluid (and structure when interactions are included) as an assemblage of large "geometric elements" or "macro-elements" that are defined by geometric entities only. Each geometric element is relatively large and consists of points, lines and surfaces, as defined by the analyst or imported from a CAD program. Given a complete geometry, there is no unique set of geometric elements, but the analyst selects these to naturally divide the complete domain. The meshing of the geometric elements is then performed by either rule-based procedures or a free meshing algorithm that is based on the advancing front method [6]. Each meshed geometric element is assigned by the analyst to an element group.

The element mesh density is prescribed by the analyst either by assigning a certain element size to a complete geometric element or different element sizes to the points, lines and surfaces defining that geometry.

It is important to recognize that the meshing is performed directly on the geometry data. The boundary conditions (prescribed velocities, temperature, tractions, etc.) are also defined for the geometry data (see Fig. 1). In the mesh generation these boundary conditions are automatically transferred to the element nodes.

The use of geometric elements is somewhat natural, in that the complete fluid and structure are represented as an assemblage of entities that individually have uniform properties and that can also be meshed effectively (that is, for which we can obtain discretizations with good element geometric properties).

If structural interactions are considered, the meshes of the structural geometric elements will in general be much coarser than those of the adjoining fluid geometric elements. The compatibility in the motions of the structural and fluid domains is maintained by the ALE formulation for the fluid and by kinematically enforcing the fluid nodal points to lie on the surface of the structural model.

\subsection{Material data}

The constitutive data of the incompressible fluid mathematical model (viscosity, conductivity, 
(a)
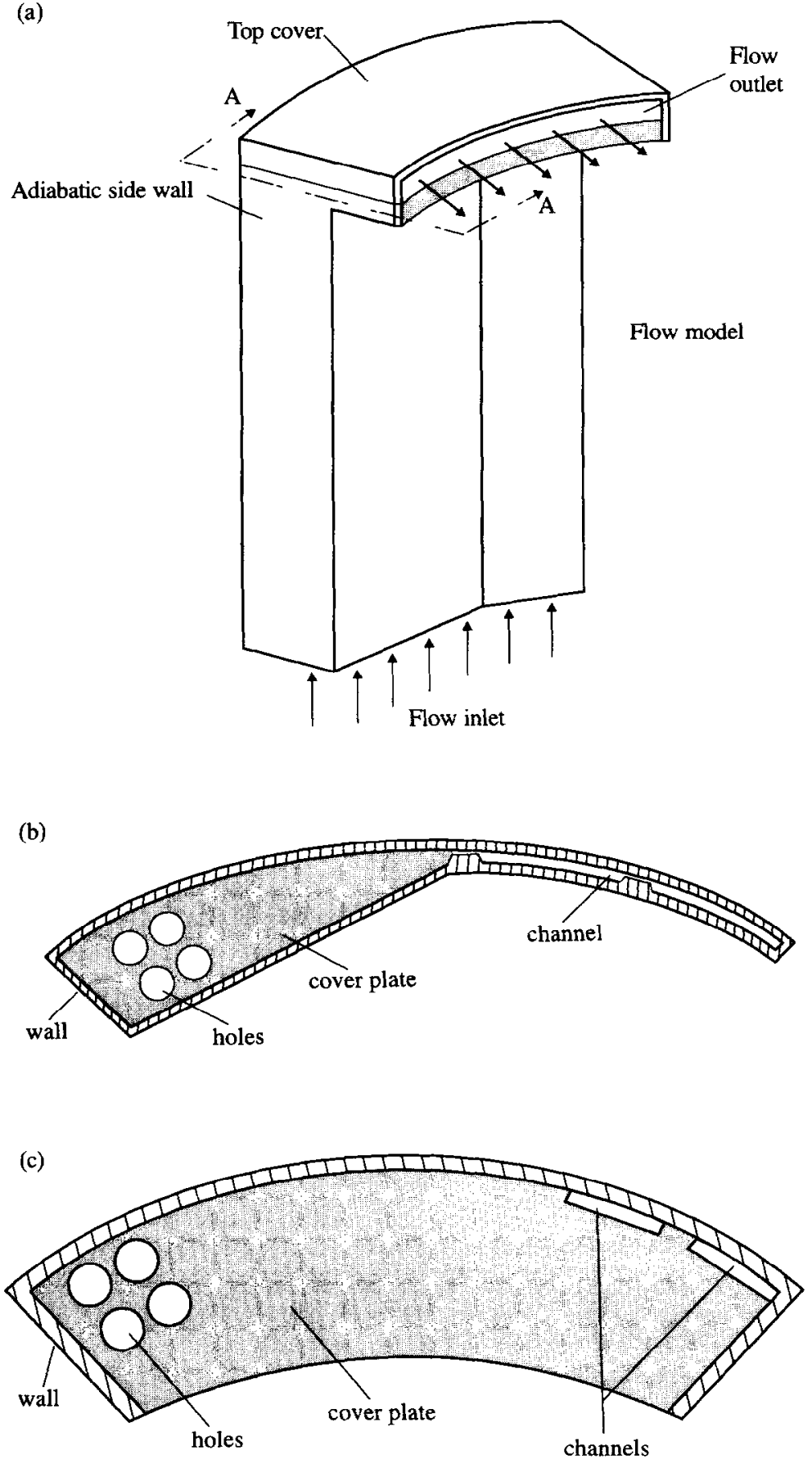

Fig. 5. $(a-c)$. Caption overleaf.

etc.) can be constant, temperature-, time- or fluidvelocity dependent.

The compressible fluid can be an ideal gas or its constitutive behavior can be defined by the general relations in eqns (22) and (23). For the use of these equations, the internal energy and mass density are defined by straight line segments as a function of pressure and temperature. The solution procedure interpolates between the straight line definitions.

\section{SOLUTION OF FINITE ELEMENT EQUATIONS}

The equations obtained for the finite element discretization are, considering transient conditions,

$$
\mathbf{M}\left(\begin{array}{c}
\hat{\hat{v}} \\
\hat{\mathbf{p}} \\
\hat{\boldsymbol{\theta}}
\end{array}\right)+\mathbf{K}(\hat{\mathbf{v}}, \hat{\mathbf{p}}, \hat{\boldsymbol{\theta}}, t)\left(\begin{array}{c}
\hat{\mathbf{v}} \\
\hat{\mathbf{p}} \\
\hat{\boldsymbol{\theta}}
\end{array}\right)=\mathbf{R}
$$

where the nodal vectors of velocities, pressure and 
(d) ADINA-F

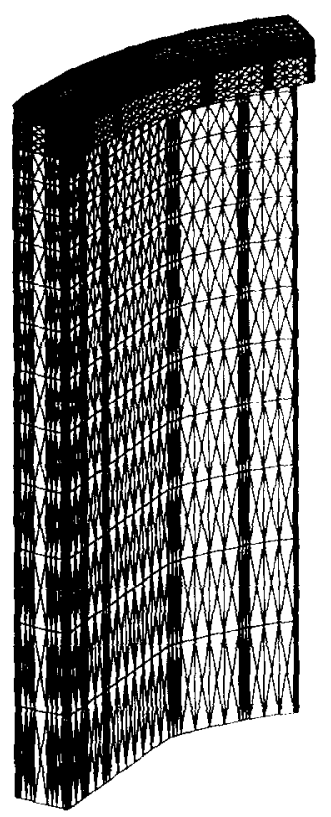

(e)

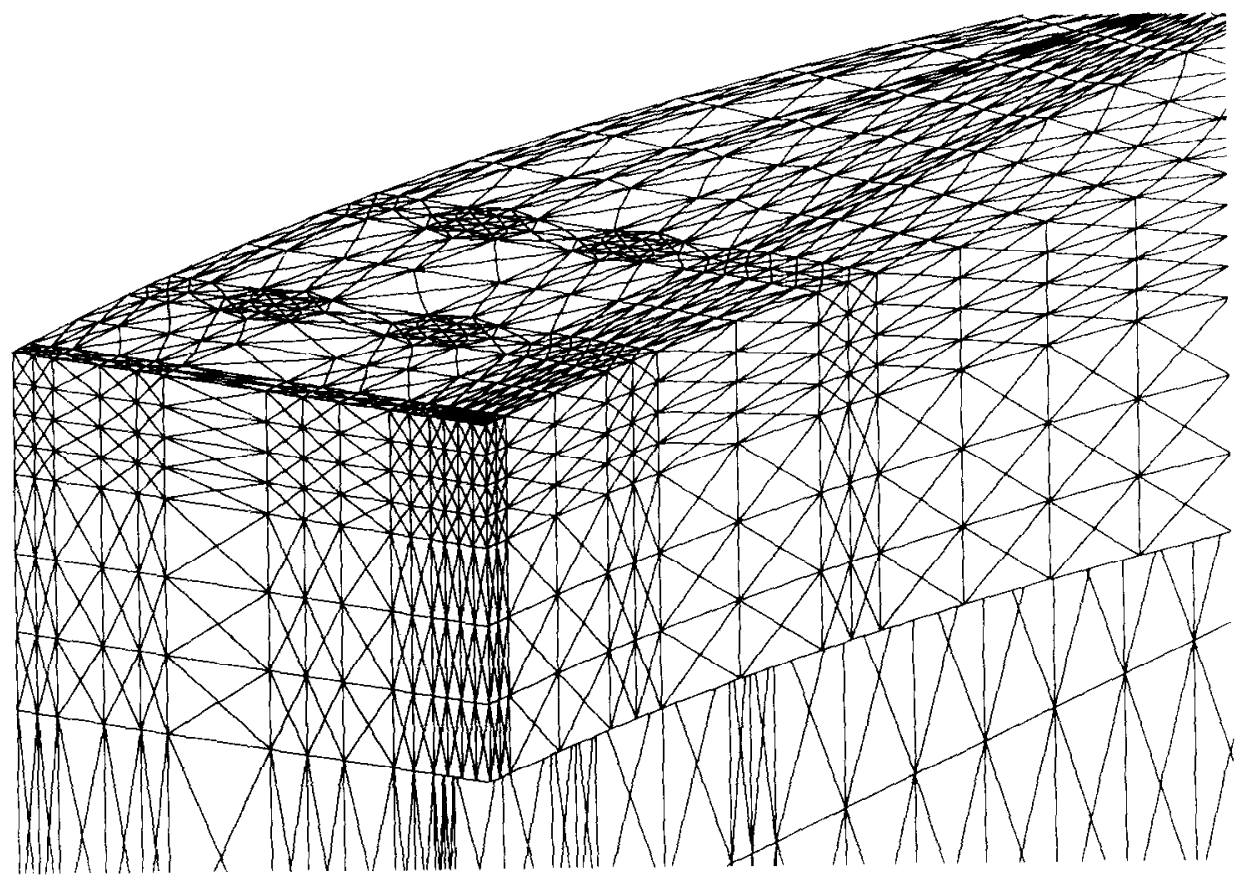

Fig. 5. Caption opposite.

temperature are $\hat{\mathbf{v}}, \hat{\mathbf{p}}$ and $\hat{\boldsymbol{\theta}}$, respectively, and $\mathbf{M}$ and $\mathbf{K}(\hat{\mathbf{v}}, \hat{\mathbf{p}}, \hat{\boldsymbol{\theta}}, t)$ are matrices corresponding to the flow conditions at time $t$.

We use the Euler methods for the time integration of eqn (32) [1]. In implicit integration of the incompressible or compressible flow equations the Euler backward method or trapezoidal rule is employed on all solution variables.

In explicit integration of the compressible fluid flow equations, all solution variables are integrated explicitly using the Euler forward method and the time step limit is

$$
\Delta t \leqslant \min _{\text {(elements) }}\left(\frac{\Delta L}{\rho\left(\tilde{\mathbf{D}}_{k}\right)}\right)
$$

where $\Delta L$ is the characteristic element side length, $\rho\left(\tilde{\mathbf{D}}_{k}\right)$ is the spectral radius of $\overline{\mathbf{D}}_{k}$, and we take the minimum over all elements. 
(f) ADINA-F
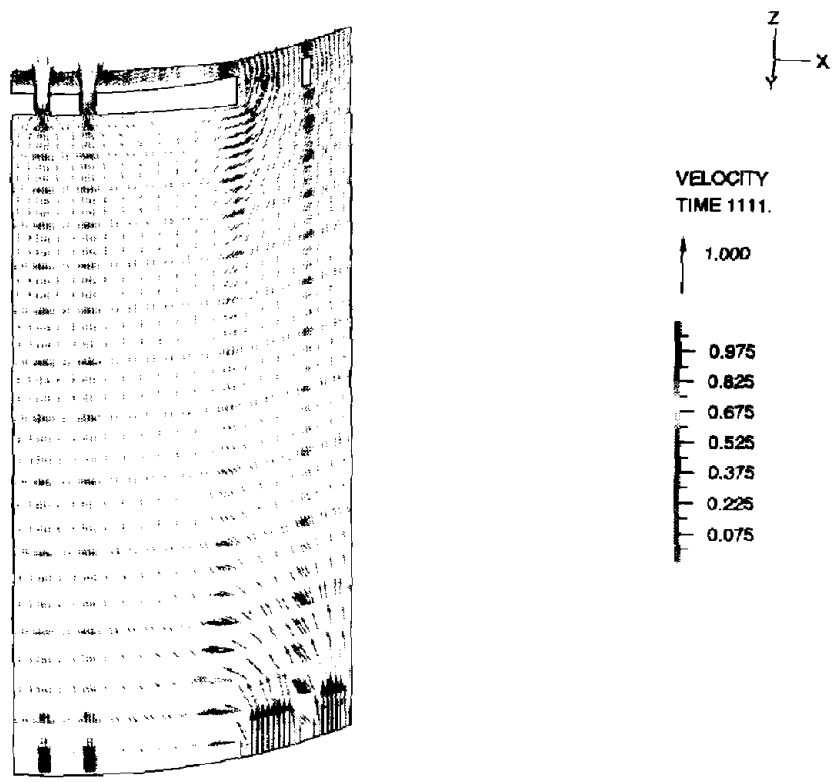

VEOCITY

TIME 1111

1.000

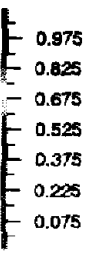

(g)

\section{ADINA-F}
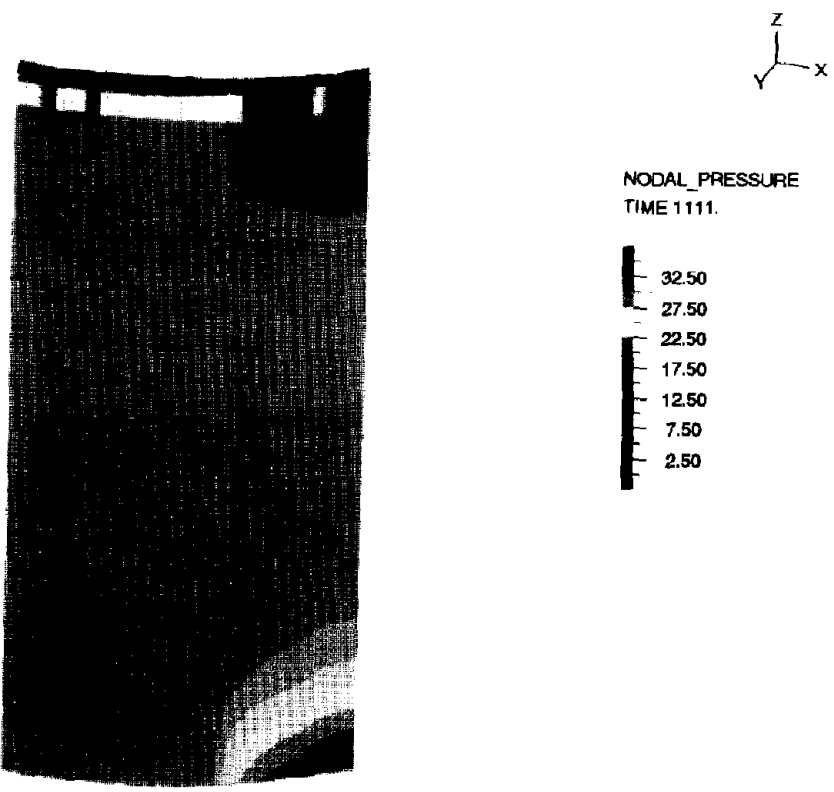

NODAL_PRESSURE TIME 1111.

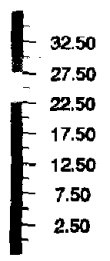

Fig. 5. Analysis of a flow device. (a) Geometry and data. (b) Inlet section. (c) Section A-A. (d) Finite element mesh used. (e) Detail of finite element mesh used (top left corner of device). (f) Predicted velocity distribution. (g) Predicted pressure distribution.

(a)

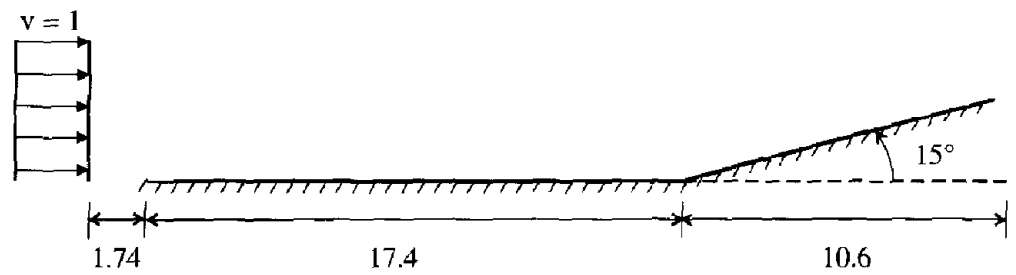

Fig. 6. (a) Caption on p. 206. 
(b)

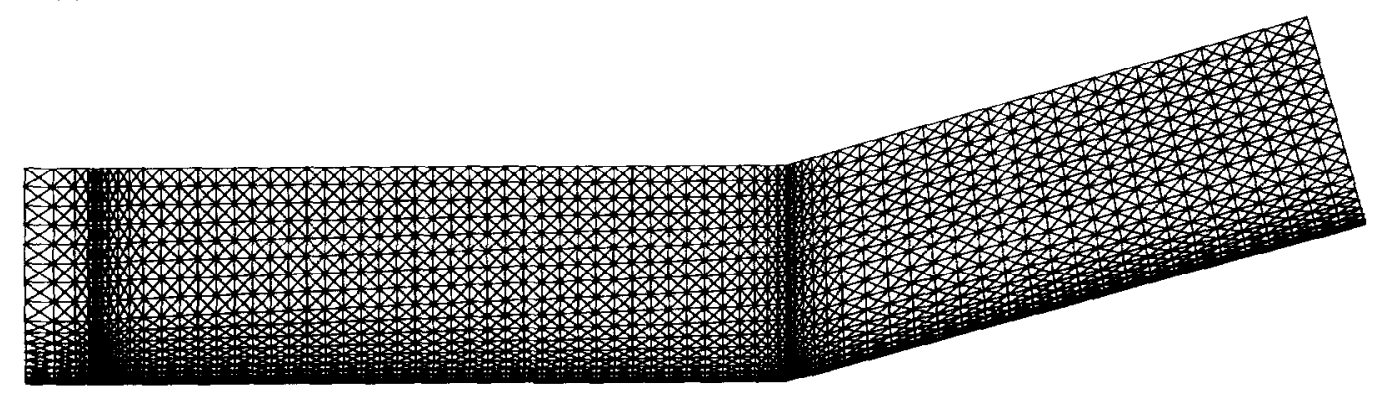

(c)

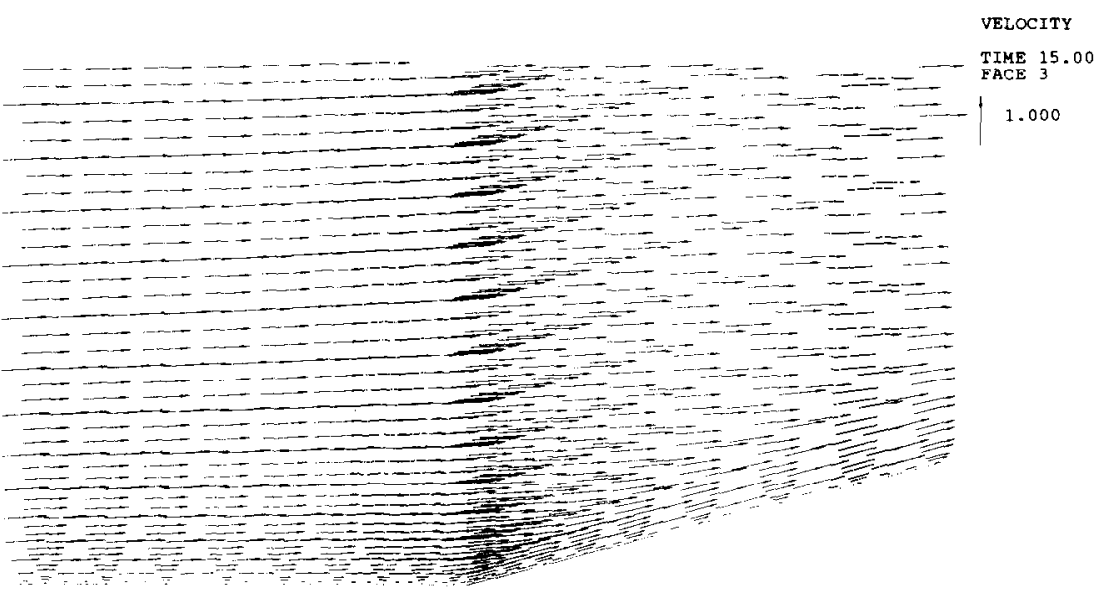

Fig. 6. (b, c) Caption on p. 206.

However, in explicit integration of the incompressible fluid flow equations, the Euler forward method is only used for the momentum and energy equations, whereas the continuity equation is still integrated implicitly. The use of implicit integration of the continuity equation is necessary to preserve the stability of the time integration and mass conservation. In this case, the time step limit is

$$
\Delta t \leqslant \min _{\text {(elements) }}\left(\frac{\Delta L}{|v|+2 \frac{v}{\Delta L}}\right)
$$

where $v$ is the velocity through the element face and $v$ is the kinematic viscosity, $v=\mu / \rho$.

In a program option, the applicable time step limit is automatically evaluated during the finite element solution and the actual time step size used is determined as the critical time step size multiplied by a user-specified factor, the CFL (Courant-Friedrichs-Lewy) number.

Of course, if implicit integration is used, the time step in the solution is only limited by the solution accuracy to be achieved and the requirement that convergence in the iterative solution of the equations must be obtained in each time step. A tangent coefficient matrix is computed and for the equation solution, the biconjugate gradient method or GMRES procedure is used with an incomplete Cholesky preconditioner [7].

To obtain the steady-state solution, implicit integration is usually used, and in this case the transient effects can be totally neglected. However, explicit integration can also be employed, in which case many time steps may be needed to reach the steady state. The advantage of using the explicit solution scheme lies in that relatively large systems can be solved for given hardware resources, because no coefficient matrix is computed.

\section{SOLUTION RESULTS OF SOME ANALYSIS PROBLEMS}

To illustrate the solution capabilities described in the previous sections, we present next the results of some analyses. All these analyses have been run using the ADINA system on engineering workstations.

\subsection{No flow test}

We consider a viscous fluid subjected to gravity loading in a rigid cavity, see Fig. 2. Zero velocities are 
(d)

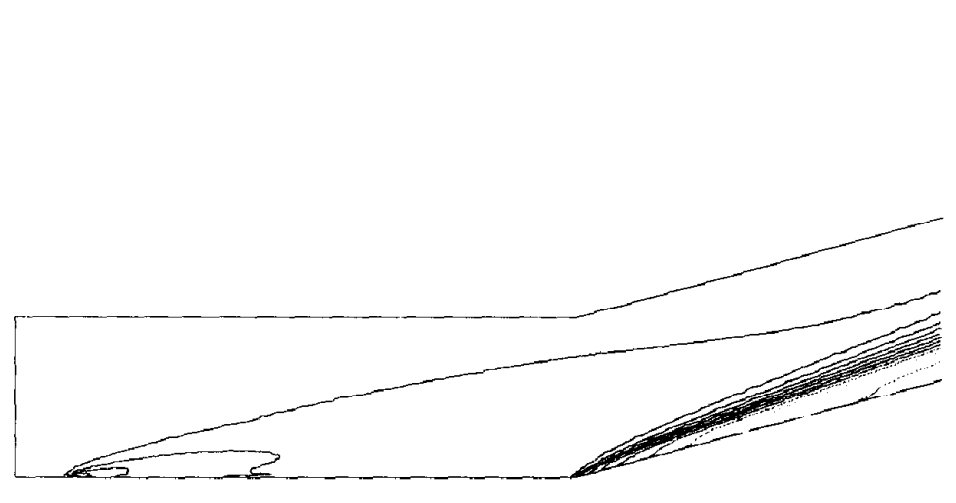

RODAL

PRESSURE

$-0.1125$

$-0.1050$

$-0.0975$

0.0900

0.0825

0.0750

0.0675

0.0600

$-0.0525$

$-0.0450$

- 0.0375

- 0.0300

$-0.0225$

$-0.0150$

$-0.0075$

(e)

TEMPERATURE

TIME 15.00

$-0.1500$

$-0.1400$

$-0.1300$

0.1200

0.1100

0.1000

0.0900

$-0.0800$

$-0.0700$

$-0.0600$

0.0500

0.0400

$-0.0300$

$-0.0200$

$-0.0100$

(f)

$-10.50$

$-\quad 9.75$

9.00

8.25

7.50

6.75

- 6.00

$-\quad 5.25$

- 4.50

3.75

3.00

$-\quad 2.25$

- 1.50

$-\quad 0.75$

Fig. 6. (d, e, f) Caption overleaf. 
(g)

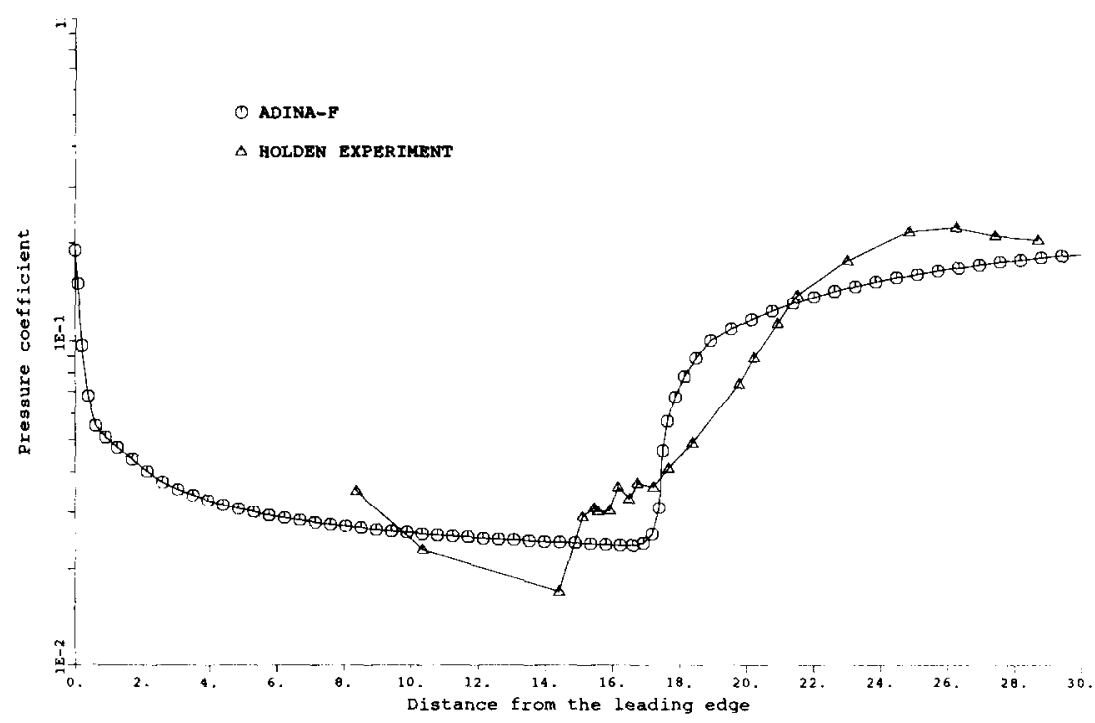

Fig. 6. Compressible fluid flow along compression corner. (a) Geometry and data; Mach number $=11.68$, Reynolds number $=248,600$. (b) Finite element mesh used. (c) Predicted velocity distribution. (d) Predicted pressure. (e) Predicted temperature. (f) Predicted Mach number distribution. (g) Comparison of pressure coefficient, predicted vs experimental results.

prescribed on the walls, but the velocities on the surface and the interior are left free. The analytical solution gives, of course, zero velocities everywhere and a linear pressure distribution. The elements used provide this analytical solution in one solution step and one iteration of a steady-state analysis (a second iteration is actually used to measure the iteration convergence).

This solution is easily obtained because the finite elentents satisfy the inf-sup condition (see Section 3.1).

\subsection{Analysis of convection of air between concentric cylinders}

The flow of air and its temperature distribution between two concentric cylinders, with the inner cylinder at a higher temperature, was analyzed, see Fig. 3(a). Experimental results have been published for this problem [8].

We used the finite element mesh shown in Fig. 3(b) and obtained the solution results given in Fig. 3(c) and (d). These solutions compare very well with the available laboratory test results [8].

\subsection{Analysis of flows in pipe connections}

The fluid flows in the pipe connections shown in Fig. 4 were solved. The objective of the analyses was to predict the flow of the fluid, and in particular the pressure drop from inlet to outlet. Figure 4(a) and (b) show a typical finite element mesh used and some solution results. Figure 4(c) shows the pressure solution results for an additional pipe connection.

\subsection{Analysis of fluid flow in a flow device}

The fluid flow in the device schematically shown in Fig. 5(a-c) has been determined. The velocity and temperature are prescribed at the inlet. Heat is supplied on the front and back walls, while the side and top walls are adiabatic. The temperatures of the structure and the fluid have been determined simultaneously by a conjugate heat transfer analysis. The finite element mesh used is given in Fig. 5(d) and (e) and Fig. 5(f) and (g) shows velocity and pressure distributions.

\subsection{Analysis of high Mach number fuid flow along plate with corner}

We analyzed the fluid flow along the compression corner shown in Fig. 6(a). The finite element mesh is given in Fig. 6(b) and Fig. 6(c-f) shows the predicted velocity, pressure, temperature and Mach number distributions. The fluid is at a Mach number of 11.68 .

These results compare quite well with those given in Ref. [9], as shown in Fig. 6(g).

\subsection{Analysis of impinging water jet}

A water jet is impinging on a rigid wall as shown in Fig. 7(a). The final shape of the jet and the flow in the jet are to be determined. We assumed axisymmetric conditions. Figure 7(b) shows the finite element mesh for an initially assumed shape of the jet. Figure 7(c) shows the finite element mesh when the jet reaches its equilibrium shape, and Fig. 7(d) and (e) give the predicted pressure and velocity distributions in the jet. 
(a)

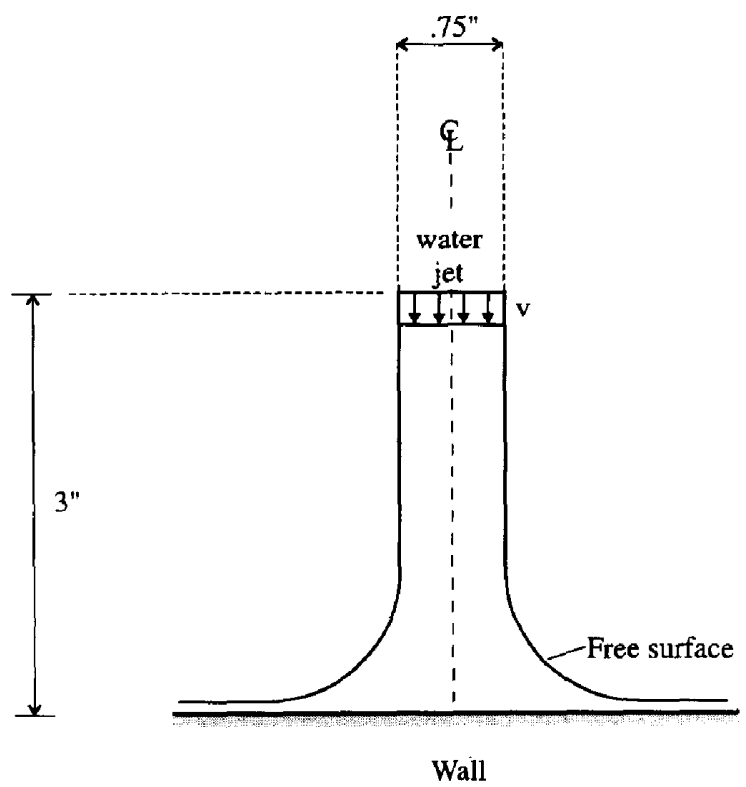

(b)

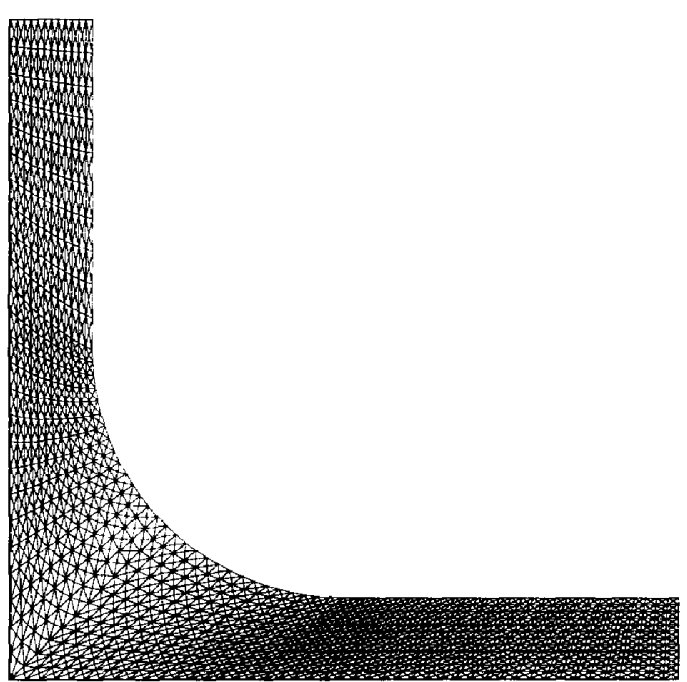

(c)

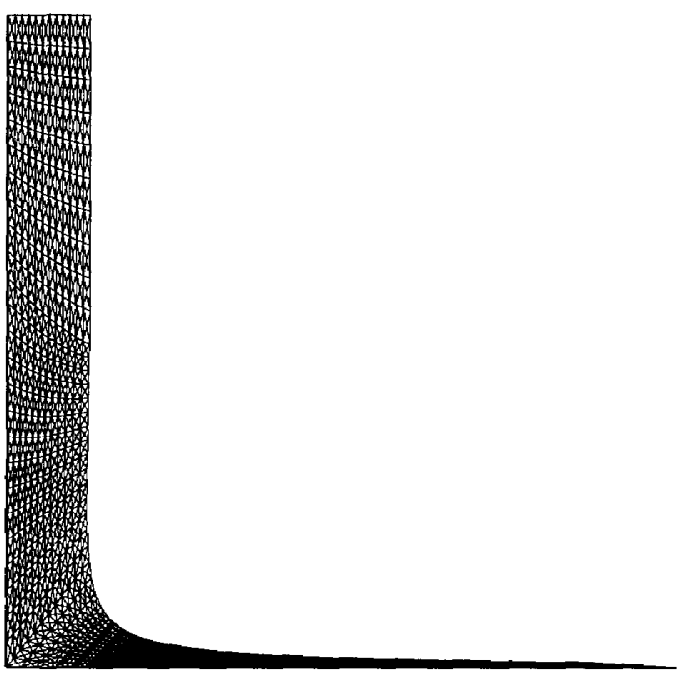

Fig. $7(\mathrm{a}, \mathrm{b}, \mathrm{c})$. Caption overleaf. 
(d)

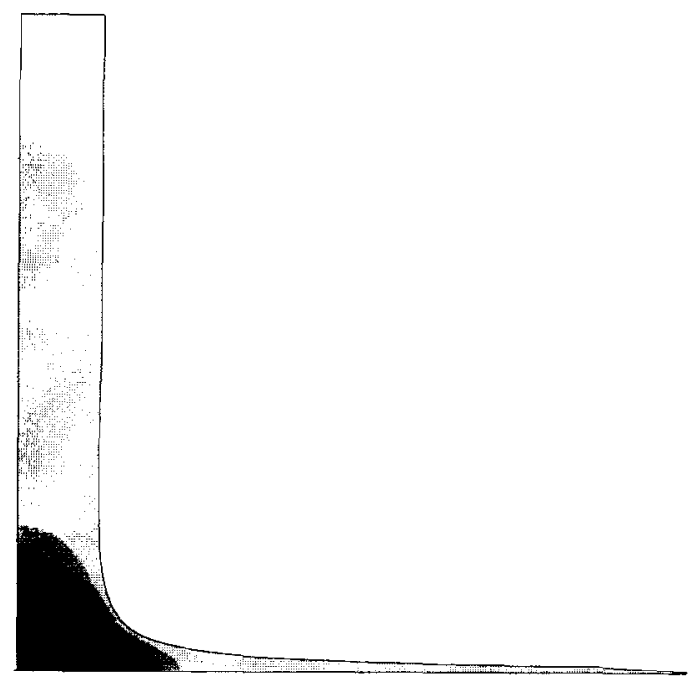

NODAL PRESSURE
TIME 0.006600

35000 .

32500 .

30000 .

27500.

25000.

22500 .

20000.

17500 .

-15000 .

-12500 .

10000 .

7500.

5000.

2500.

o.

(e)

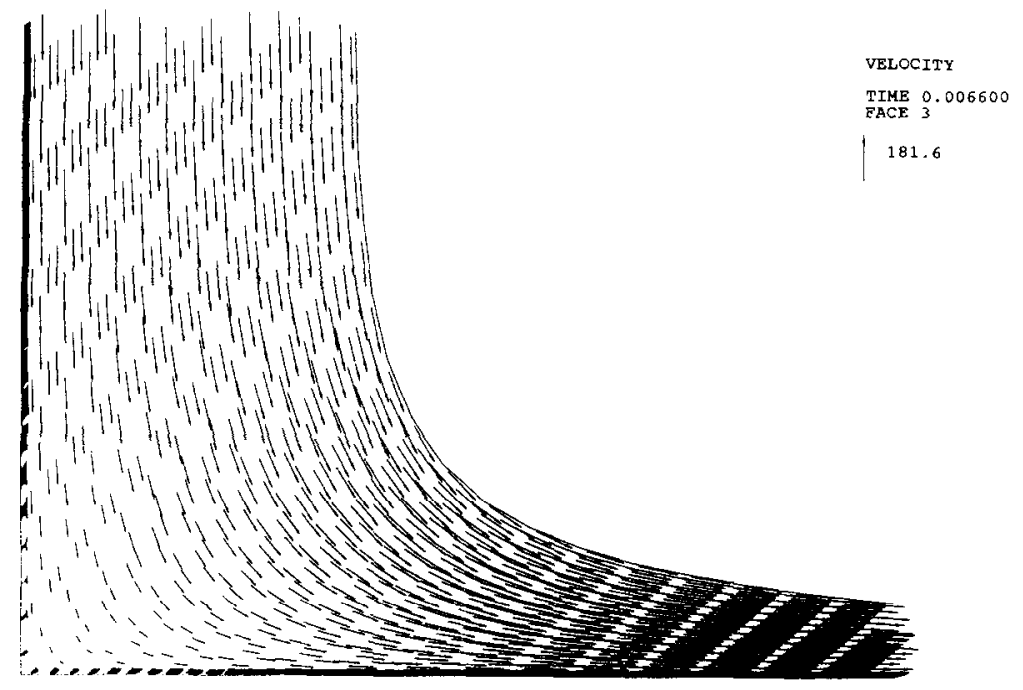

Fig. 7. Analysis of an impinging water jet. (a) Geometry and data. (b) Initially assumed geometry (also shows finite element mesh used). (c) Finite element mesh at flow equilibrium of jet. (d) Predicted pressure distribution. (e) Predicted velocity distribution.

\subsection{Analysis of parachute "free-fall" conditions}

We analyzed the fluid-structure interaction problem of a parachute falling in steady-state conditions, as schematically shown in Fig. 8(a). Assuming axisymmetric conditions, we used the structural model shown in Fig. 8(b).

Some solution results are given in Fig. 8(c) and (d). The velocity and pressure distributions correspond to conditions that we should expect, see Ref. [10].
5.8. Analysis of container subjected to fluid-flow loading

The container shown in Fig. 9(a) is a thin shell structure loaded by the fluid flow. We used the meshes shown in Fig. 9(b) and (c), respectively, for the structure and the fluid. Note that the finite elements representing the structure are much larger than those representing the fluid. 
(a)

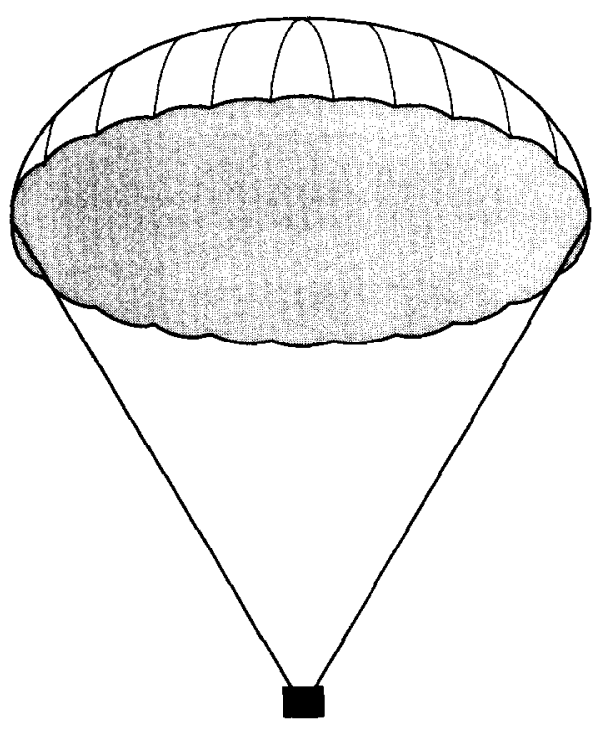

(b)

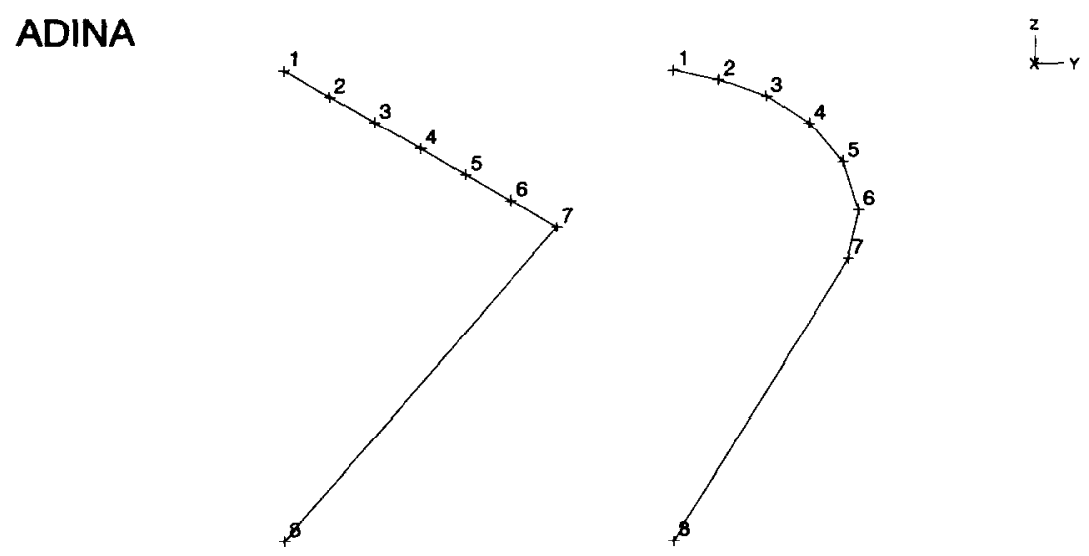

Fig. 8. (a, b) Caption overleaf.

Figures 9(d)-(f) show some solution results. As seen, the fluid flow causes relatively large deformations in the shell container.

\section{CONCLUDING REMARKS}

We have briefly surveyed some solution capabilities for general fluid flow analyses. These capabilities represent a powerful tool but, of course, the field of computational fluid dynamics (CFD) is rapidly progressing and continuous further developments are needed. These developments pertain, for example, to the efficient use of self-adaptive techniques and the implementation of the solution procedures on parallel processing machines. We are pursuing these and other developments and see an exciting future for the application of our CFD procedures. 
(c)

\section{ADINA-F}
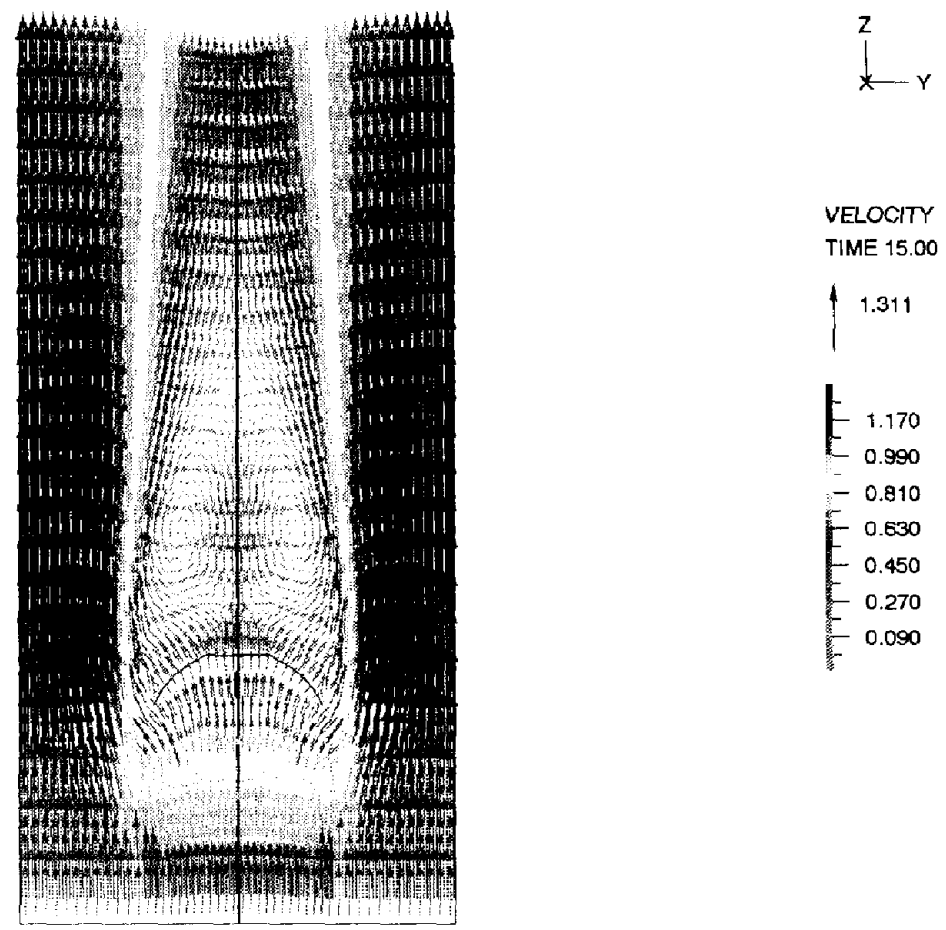

VELOCITY

TIME 15.00

1.311

1.31

1.170
0.990

- 0.810

- 0.630

- 0.450

0.270

0.090

(d)

\section{ADINA-F}
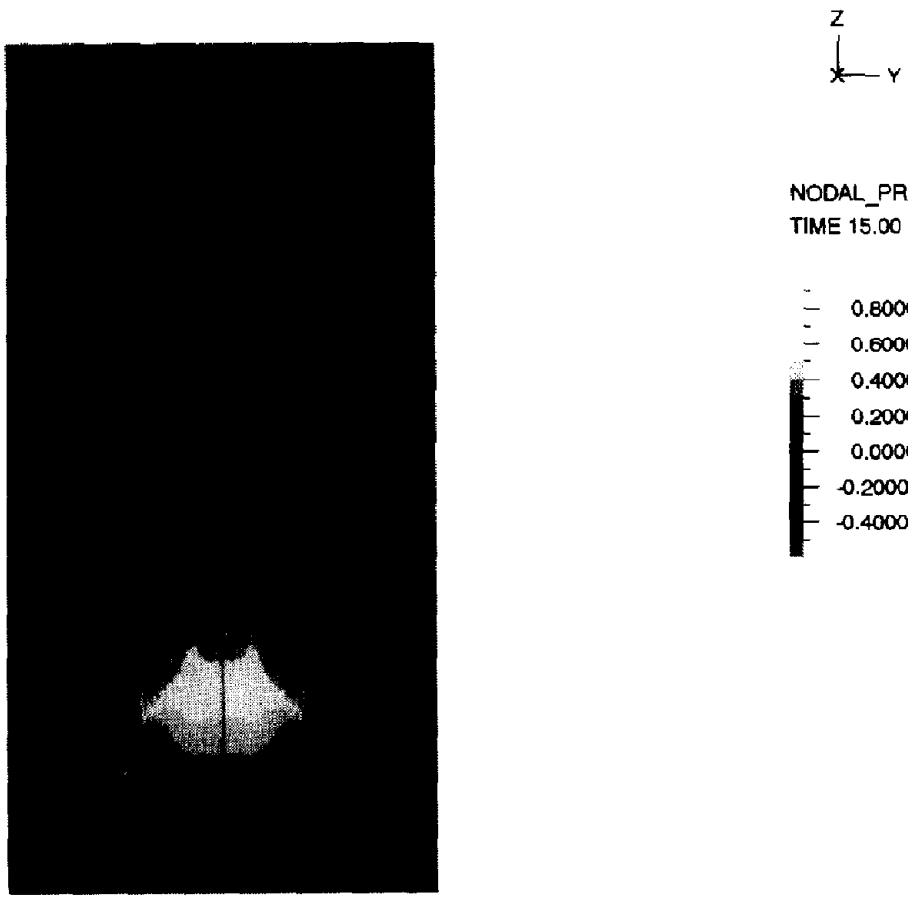

NODAL_PRESSURE

TIME 15.00

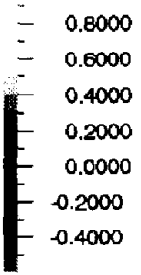

Fig. 8. Analysis of a freely falling parachute (a) Geometry (b) Finite element mesh of structure, in initial configuration and deformed configuration. (c) Predicted air flow. (d) Predicted pressure distribution. 
(a)

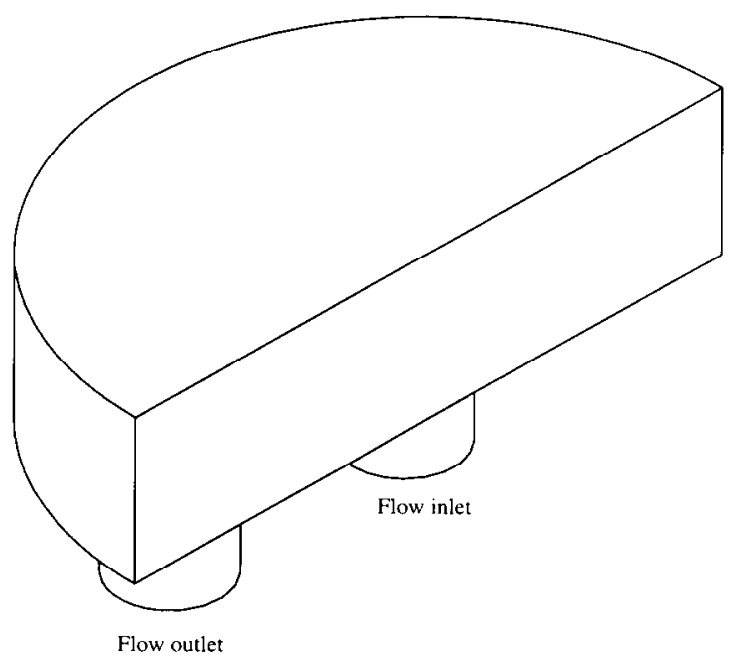

(b) ADINA

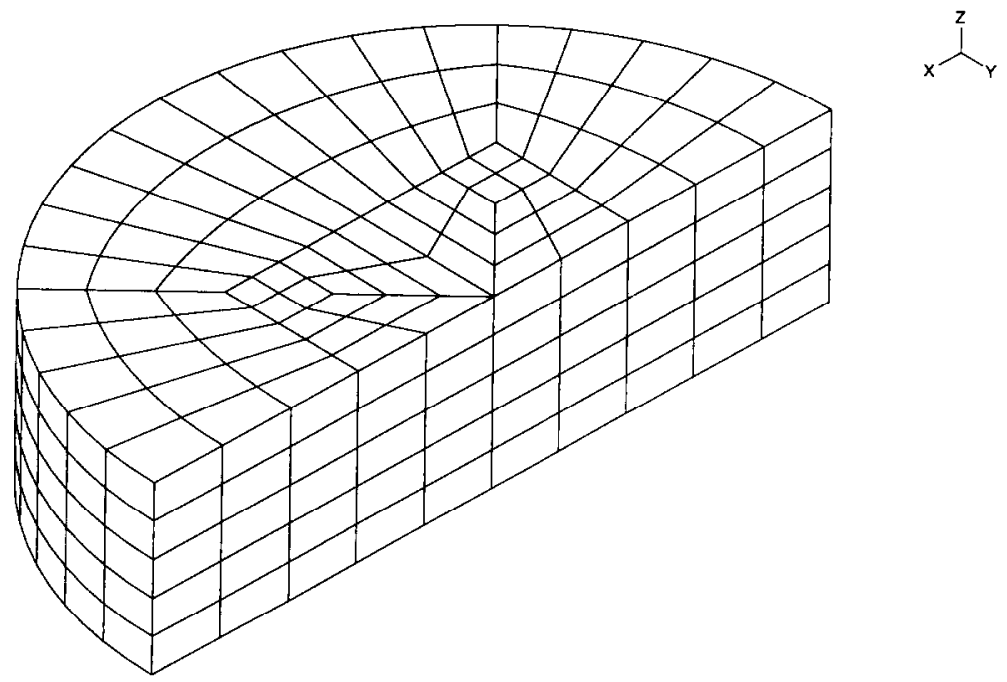

(c) ADINA-F

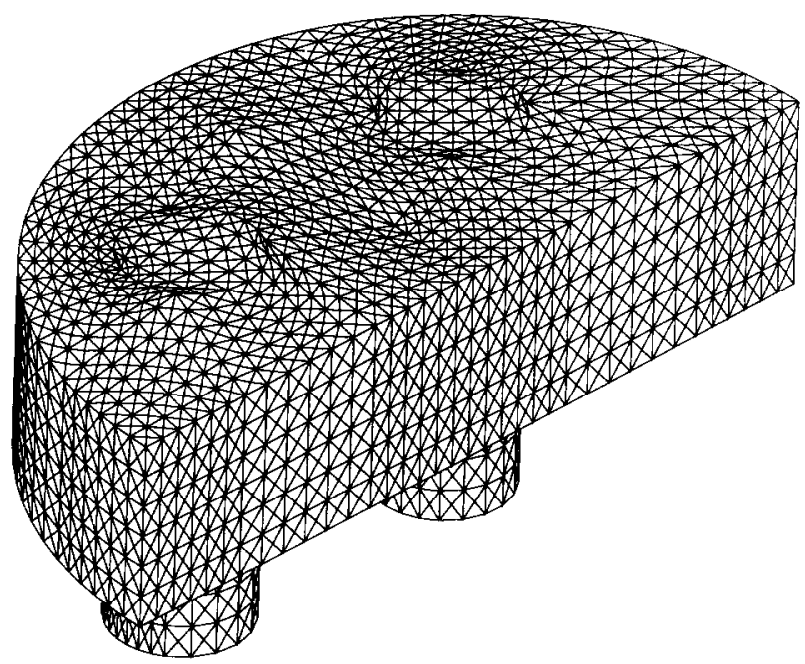

$\mathcal{L}_{x}^{z}$

Figures 9. $(\mathrm{a}-\mathrm{c})$ Caption overleaf. 
(d) ADINA

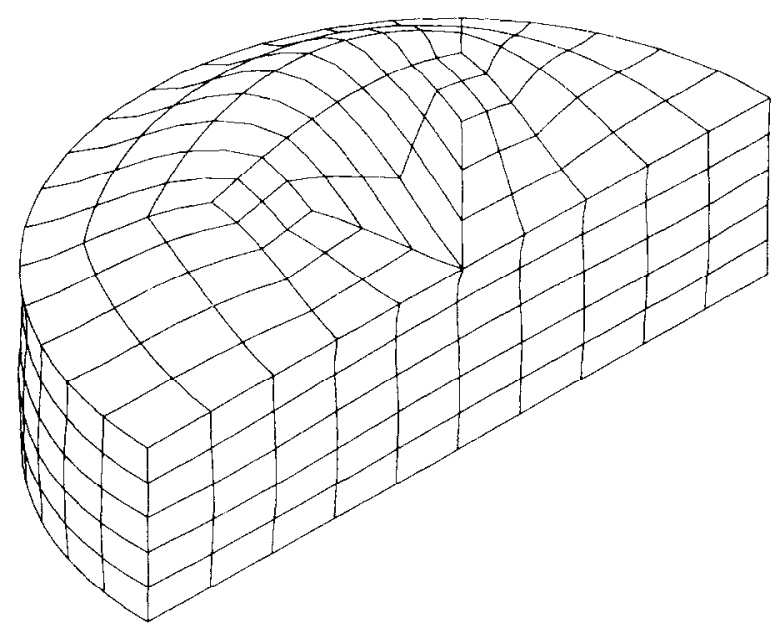

$\lambda^{2}$

(e)

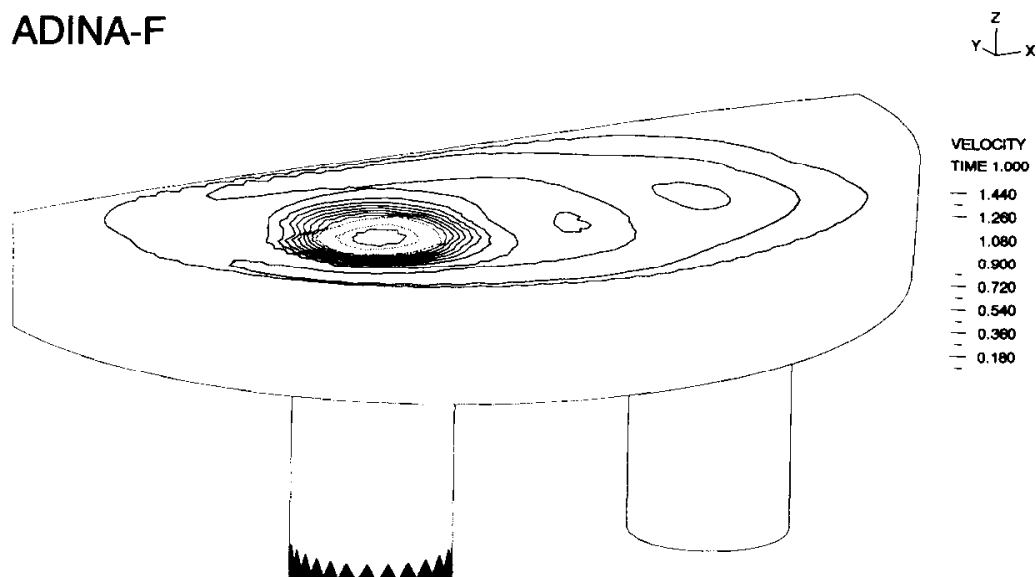

(f)

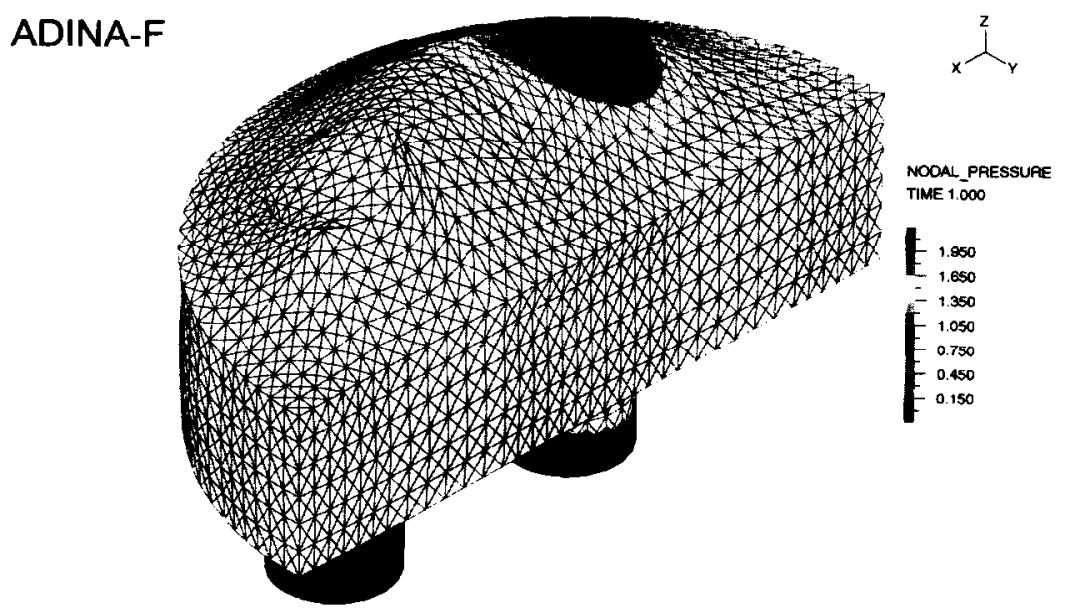

Fig. 9. Analysis of container subjected to fluid flow loading. (a) Geometry. (b) Mesh of eight-node shell elements used for container. (c) Mesh used for fluid in container. (d) Deformed structural mesh (deformations are drawn to the same scale as the structure). (e) Predicted velocities in fluid. (f) Predicted pressure in fluid. 


\section{REFERENCES}

1. K. J. Bathe, Finite Element Procedures. Prentice Hall, Englewood Cliffs, NJ (1995).

2. J. Donea, S. Giuliani and J. P. Halleux, An arbitrary Lagrangian-Eulerian finite element method for transient dynamic fluid-structure interactions. Comput. Meth. appl. mech. Engng 33, 689-723 (1982).

3. C. Nitikitpaiboon and K. J. Bathe, An arbitrary Lagrangian-Eulerian velocity potential formulation for fluid-structure interaction. Comput. Struct. 47(4,5), 871-891 (1993).

4. D. Chapelle and K. J. Bathe, The inf-sup test. Comput. Struct. 47(4, 5), 537-545 (1993).

5. H. Zhang, J. Y. Trépanier, M. Reggio and R. Camarero, A Navier-Stokes solver for stretched triangular grids, paper AIAA92-0183, 30th Aerospace Sciences Meeting, Reno, NV (1992).
6. K. Morgan, J. Peraire, J. Peiro and O. Hassan, The computation of three-dimensional flows using unstructured grids. Comput. Meth. appl. Mech. Engng 87, $335-352$ (1991).

7. L. H. Tan and K. J. Bathe, Studies of finite element procedures - the conjugate gradient and GMRES methods in ADINA and ADINA-F. Comput. Struct. 40(2), 441-449 (1991).

8. M. Van Dyke, An Album of Fluid Motion. The Parabolic Press (1982).

9. M. S. Holden, A study of flow separation in regions of shock wave-boundary layer interaction in hypersonic flow. AIAA 11th Fluid and Plasma Dynamics Conf., Seattle, Washington (1978).

10. K. R. Stein and R. J. Benney, Parachute inflation: a problem in aeroelasticity, Technical Report NATICK/TR-94/015, United States Army (1994). 